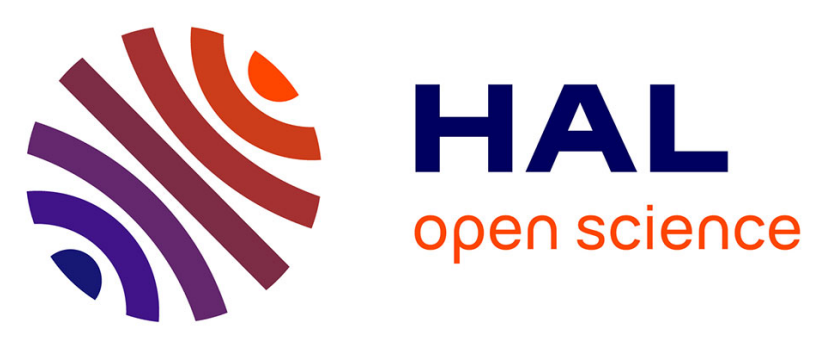

\title{
Wearable and Fully-Portable Smart Garment for Mechanical Perturbation Detection with Nanoparticles Optical Fibers
}

Arnaldo G. Leal-Junior, Diana Ribeiro, Leticia M. Avellar, Mariana Silveira, Camilo A. Rodriguez Díaz, Anselmo Frizera-Neto, Wilfried Blanc, Eduardo Rocon, Carlos Marques

\section{To cite this version:}

Arnaldo G. Leal-Junior, Diana Ribeiro, Leticia M. Avellar, Mariana Silveira, Camilo A. Rodriguez Díaz, et al.. Wearable and Fully-Portable Smart Garment for Mechanical Perturbation Detection with Nanoparticles Optical Fibers. IEEE Sensors Journal, 2021, 21 (3), pp.2995-3003. 10.1109/JSEN.2020.3024242 . hal-03137161

\author{
HAL Id: hal-03137161 \\ https://hal.science/hal-03137161
}

Submitted on 10 Feb 2021

HAL is a multi-disciplinary open access archive for the deposit and dissemination of scientific research documents, whether they are published or not. The documents may come from teaching and research institutions in France or abroad, or from public or private research centers.
L'archive ouverte pluridisciplinaire HAL, est destinée au dépôt et à la diffusion de documents scientifiques de niveau recherche, publiés ou non, émanant des établissements d'enseignement et de recherche français ou étrangers, des laboratoires publics ou privés. 


\title{
Wearable and Fully-Portable Smart Garment for Mechanical Perturbation Detection With Nanoparticles Optical Fibers
}

\author{
Arnaldo G. Leal-Junior ${ }^{\circledR}$, Member, IEEE, Diana Ribeiro, Leticia M. Avellar, Mariana Silveira, \\ Camilo A. Rodriguez Díaz ${ }^{\circledR}$, Member, IEEE, Anselmo Frizera-Neto ${ }^{\circledR}$, Member, IEEE, \\ Wilfried Blanc ${ }^{(1)}$, Eduardo Rocon, Member, IEEE, and Carlos Marques ${ }^{(1)}$
}

\begin{abstract}
This paper presents the development of a wearable smart textile for mechanical perturbation assessment during gait based on distributed optical fiber sensor system using the transmission-reflection analysis (TRA). In this case, magnesium and erbium co-doped optical fiber is employed as a high scattering medium, which results in high spatial resolution for the technique (in the order of a few millimeters). The optical fiber was embedded in a garment and controlled displacements at predefined locations on the garment were applied, where the simultaneous assessment of displacement amplitude and location was obtained using a slope-assisted technique. In the proposed technique, the slope inverses of the transmission-reflection curves are correlated with the displacement applied on the fiber, where a determination coefficient $\left(R^{2}\right)$ of 0.996 was obtained. Then, the sloped-normalized reflection (or backscattered) response is used on the disturbance location estimation with relative errors as low as $3.7 \%$. The on-body application tests show an inherent insensitivity of the proposed smart garment to body movement due to natural gait movements, indicating the feasibility of the proposed approach on detecting only the transverse induced mechanical perturbations. In the wearable tests, the sensor system shows high correlation $\left(R^{2}\right.$ higher than 0.98 ) in the tests with different volunteers (subjected to similar displacements at predefined locations) and millimeter-accuracy on the disturbance location estimation.
\end{abstract}

Index Terms-Distributed optical fiber sensors, gait perturbation, transmission-reflection analysis, particle-containing glasses.

Manuscript received August 15, 2020; revised September 9, 2020; accepted September 9, 2020. Date of publication September 15, 2020; date of current version January 6, 2021. This work was supported in part by Fundação de Amparo à Pesquisa e Inovação do Espírito Santo (FAPES) under Grant 84336650 ; in part by the National Council for Scientific and Technological Development (CNPq) under Grant 304049/2019-0 and Grant 408480/2018-1; in part by Petrobras under Grant 2017/00702-6, in part by the national funds (OE), through FCT, I.P., "in the scope of the framework contract foreseen in the numbers 4,5 and 6 of the article 23, of the Decree-Law 57/2016, of August 29, changed by Law $57 / 2017$, of July 19:" in part by the Agence Nationale de la Recherche (ANR) under Grant ANR-17-CE08-0002; and in part by the BenchBalance European Union (EU) Project under Grant EUH2020-779963-FSTP1-BenchBalance. The work of Carlos Marques was supported in part by the Fundação para a Ciência e a Tecnologia (FCT) through the (iFish project) under Grant CEECIND/00034/2018 and in part by the national funds through the FCT/MEC through the scope of the project i3N under Grant UIDB/50025/2020 and Grant UIDP/50025/2020. The associate editor coordinating the review of this article and approving it for publication was Dr. Daniele Tosi. (Corresponding author: Arnaldo G. Leal-Junior.)

Arnaldo G. Leal-Junior, Mariana Silveira, Camilo A. Rodriguez Díaz, and Anselmo Frizera-Neto are with the Graduate Program of Electrical Engineering of Federal University of Espirito Santo, Vitória 29075-910, Brazil (e-mail: leal-junior.arnaldo@ieee.org; marianalyrasilveira@ gmail.com; c.rodriguez.2016@ieee.org; frizera@ieee.org).
Diana Ribeiro and Carlos Marques are with the I3N \& Physics Department, Universidade de Aveiro, Campus Universitário de Santiago, 3810-193 Aveiro, Portugal (e-mail: diana.reis.ribeiro14@live.ua.pt; carlos.marques@ua.pt).

Leticia M. Avellar is with the Graduate Program of Electrical Engineering of Federal University of Espirito Santo, Vitória 29075-910, Brazil, and also with the Centro de Automática y Robótica, CSIC-UPM, 28500 Arganda del Rey, Spain (e-mail: leticia.avellar@aluno.ufes.br).

Wilfried Blanc is with the Institut de Physique de Nice (INPHYNI), UMR 7010, Université Côte d'Azur, 06108 Nice, France (e-mail: wilfried. blanc@inphyni.cnrs.fr).

Eduardo Rocon is with the Centro de Automática y Robótica, CSIC-UPM, 28500 Arganda del Rey, Spain (e-mail: e.rocon @ csic.es).

Digital Object Identifier 10.1109/JSEN.2020.3024242

\section{INTRODUCTION}

$\mathbf{I}$ MPACT detection and pressure mapping play an important role in different application fields from automotive to structural health monitoring in civil structures as well as mechanical components [1]. In addition, the pressure mapping is an important tool and is commonly used in conjunction with the impact detection for the assessment of structural damage, identification of stress concentration due to errors in project 
or material fatigue [2]. The pressure mapping is also important for biomechanical applications such as plantar pressure mapping [3], human-robot [4] and human-environment [5] interactions.

The investigation on human balance during walking has been a research interest for many years, where center of mass and center of pressure investigations under different standing and gait conditions are reported [6]. In this context, the gait analysis under mechanical perturbations provides important insight on human balance control, including the spatiotemporal parameters and dynamic stability in gait [7]. The neuromuscular activity on forward and backward perturbations during gait was also measured, where the magnitude and location of the perturbation can lead to different muscular responses [8]. Thus, the detection of the location and amplitude of the perturbation provide additional information regarding the natural strategies of balance control in humans.

The widespread of wearable robots for gait assistance [9] points towards the development of bioinspired devices from the design with approaches such as the human-in-the-loop design [10] to the device control [11], including bioinspired actuation [12]. The investigation of the human balance control can lead to the development of novel robust control strategies for wearable robots with disturbance rejection [13]. In addition, experimental protocols for mechanical disturbances during exoskeleton-assisted gait provide quantitative and qualitative data regarding the exoskeleton control robustness, which also indicates the importance of a wearable impact detection system in biomechanical and health monitoring applications.

For impact detection systems, electronic sensors based on capacitive and resistive technologies are commonly used [14]. Recently, the developments of conductive polymers also resulted in pressure sensors embedded in textiles [15]. However, the electromagnetic field sensitivity, lack of multiplexing capabilities as well as the sensor wiring are significant drawbacks in these systems especially when their use with wearable robots is proposed [16]. To that extent, optical fiber sensors have been proposed through the years, since they rely on advantages such electromagnetic field immunity, chemical stability, galvanic isolation as well as compactness and multiplexing capabilities [17]. In addition to industrial and structural health monitoring applications, the advantages of optical fiber sensors motivate their applications in healthcare [18], including on the wearable robots instrumentation [16].

In impact (or perturbation) detection systems using optical fiber sensor technologies, the distributed [19] and quasidistributed [20] approaches are employed for disturbances location assessment in a kilometer range. Distributed optical fiber sensors are mainly based on optical time-domain reflectometry (OTDR) and optical frequency-domain reflectometry (OFDR) with spatial resolution on the ranging from centimeters to meters [19]. Although such spatial resolution is proportionally high when sensing lengths in the order of kilometers are considered, such spatial resolution in order of meters is regarded as a low spatial resolution in systems with sensing lengths as small as 8 meters, for example. In addition, such approaches have a complex interrogation system with bulk and costly components such as modulated light sources and, in some cases, microwave photonic circuits [19]. Such systems can also present acquisition rate of a few samples per second, which inhibit their application in dynamic events assessment.

In contrast, quasi-distributed approach is generally based on fiber Bragg gratings (FBGs), where the spatial resolution is limited by the physical separation between FBGs, which can be as low as a few centimeters [21]. However, most of commercially available FBG interrogators are bulk and nonportable. For this reason, special interrogators such as the one proposed in [22] are needed for wearable applications of such sensor systems. In addition, specialized equipment is needed for the FBG array inscription in the optical fiber, which also leads to structural modification in the optical fiber.

As an alternative approach for distributed optical fiber sensors, the transmission-reflection analysis (TRA) has been proposed [23]. In this case, the transmitted and reflected optical powers are measured and correlated with the location and amplitude of mechanical perturbations on the optical fiber [24]. Therefore, a distributed sensor system is obtained with portable and low cost components, which is suitable for mechanical perturbation detection in wearable applications. Although there are many reports of TRA-based distributed sensors for parameters such as leak localization (through the mechanical disturbances in the optical fiber due to polymer swelling) [25], intrusion detection [26] and strain sensing [24], most of the reports are dated back to the early 2000s. The reason for this more than 10 years' gap can be related to the poor spatial resolution of the sensor (in the order of meters). Such low spatial resolution is due to the low Rayleigh scattering coefficient of standard single mode fibers (SMFs), which result in a low backscattered optical signal. However, a novel optical fiber was recently proposed [27] in which the fiber core is composed of silica and sub-200 nm oxide nanoparticles. In this optical fiber, there is an increase of the Rayleigh scattering (when compared with standard SMFs), which results in a higher backscattered optical power, leading to an increase in the spatial resolution of TRA-based systems [28]. The fibers are prepared using Modified Chemical Vapor Deposition (MCVD) process and they are drawn in standard conditions which results on the possibility of fabricating longer lengths of the fiber with competitive cost. The use of this nanoparticle optical fiber (NPF) was already demonstrated for the multiplexed sensors systems in optical backscatter reflectometer [29], [30]. In addition, FBGs were inscribed in this fiber for polarization dependent temperature sensors [31].

Considering this background in conjunction with the widespread of optical fiber sensors-based smart textiles, this paper presents the development of a NPF-embedded smart garment for mechanical perturbation assessment. The sensor is based on the TRA technique applied in the NPF, where spatial resolution in the order of a few millimeters can be achieved. It is important to mention that although polymer optical fibers (POFs) have larger strain limits and fracture toughness than the NPF (based on silica fiber), POFs does not have a high 
scattering medium such as the one of the NPF. Therefore, the millimeter-scale spatial resolution will not be reached if POFs are used. In addition, a new approach for the transmission and reflection signals processing is proposed in which the simultaneous assessment of the perturbation location and fiber displacement (as well as strain, stress and force) can be achieved. The proposed sensor system is low-cost, portable and capable of detecting dynamic events, which motivate its application on the assessment of mechanical perturbations. The proposed application was validated in different volunteers and in different scenarios, i.e., standing and walking.

\section{Sensor Development and Experimental Setup}

In the fiber fabrication (detailed description can be found in [27], [32]), there is the preparation of the silica preform with nanoparticles with their formation triggered by the use of magnesium $(\mathrm{Mg})$, an alkaline earth element, in conjunction with erbium ions dispersed in an ethanol solution to obtain the doping solution [27]. In the first step, a germanium-doped silica porous layer is obtained in Modified Chemical Vapor Deposition process. Then, it is immersed in the solution and dried at high temperature $\left(1000^{\circ} \mathrm{C}\right)$, represented in Fig. 1(a) as step 2. After thermal treatments, the porous layer is densified and resulted in a preform with $10 \mathrm{~mm}$ diameter with $1 \mathrm{~mm}$ core [27], in step 3. Following this procedure, there is an immiscible system, which results in one phase with silica predominance in the core of preform and one phase majorly composed by the Mg-based nanoparticles [27]. Finally, in step 4 , the optical fiber takes its final shape using a draw tower with $2200{ }^{\circ} \mathrm{C}$, resulting in a total diameter of $125 \mu \mathrm{m}$ of the fiber. As a final step in the fiber fabrication, a polymer coating is applied to increase the mechanical robustness of the optical fiber, especially against bending and impact, which is important for the proposed application, where the fiber is subjected to sequential mechanical perturbations. It is worth noting that the nanoparticle composition depends on its size, also resulting in a refractive index change on the particles [27]. The size of nanoparticles ranges from few nanometers to $160 \mathrm{~nm}$ with variations in shape as well. These unique characteristics of the NPF points to the necessity of a mechanical perturbations characterizations at different positions in the optical fiber prior to the sensor's application in perturbation assessment during gait. The fabrication process is summarized in Fig. 1(a), where the fabricated fiber has an attenuation of $2.6 \mathrm{~dB} / \mathrm{m}$ and a scattering gain of $2.0 \mathrm{~dB}$, which make it suitable for the transmission of optical signals in the order of $10 \mathrm{~mW}$ for a few meters. Comparing with the MgO-based nanoparticle fibers previously reported [33], [34], in this case, the fiber has an outer ring with the $\mathrm{MgO}$-based nanoparticles, which resulted in smaller optical attenuation than the ones with MgO-based nanoparticles inside the fiber's core [34].

Furthermore, based on the immiscible system created on the fiber fabrication, the interaction between the optical signals and the nanoparticles mostly occurs in the mode evanescent field. This behavior is especially desirable for distributed sensors using TRA, where there is an increase on the backscattered optical power without high transmission losses. It is well known that mechanical disturbances, such as bending, lead to

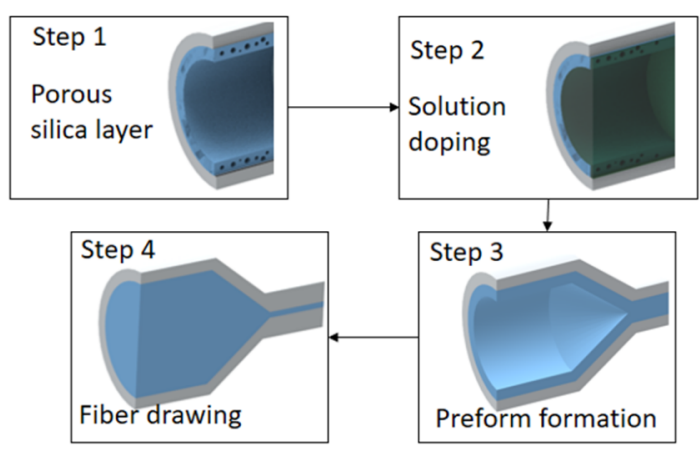

(a)

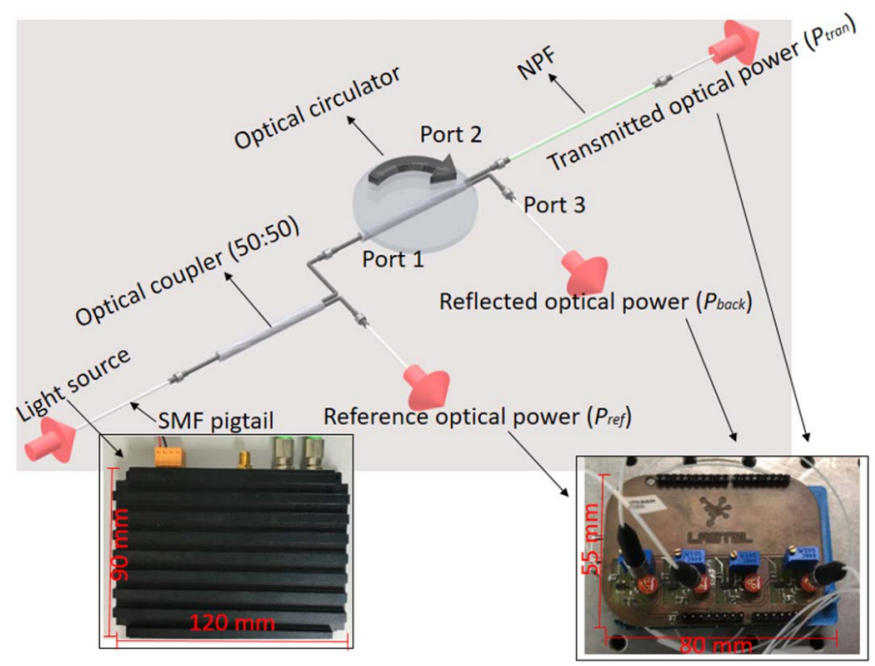

(b)

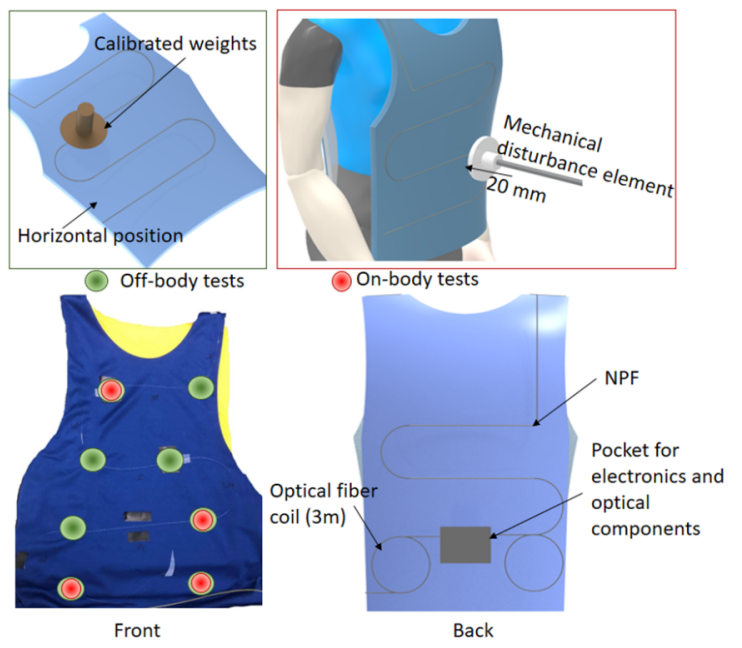

(c)

Fig. 1. (a) Schematic representation of the steps followed in the fiber fabrication. (b) Schematic representation of the optical components for the proposed distributed sensor system. Figure insets show the photographs of the light source and the electronics. (c) Experimental setup (for off- and on-body tests) and NPF positioning in the smart garment. Inset shows the fiber displacement under mechanical perturbation.

variations on the transmitted optical power and such variations are proportional to the bending angle or momentum [35]. Such mechanical disturbances also influence the backscattered optical power, which is commonly used in distributed 
sensing for estimating the disturbance location [23]. For the mechanical disturbance localization using TRA, the ratio between transmitted and reflected optical powers are employed and their unique relation at each disturbance location and amplitude result in the disturbance location estimation. In this case, the disturbance location along the fiber can be achieved with unmodulated light source, which reduces the system cost, when compared with the reflectometry-based distributed systems [24].

In TRA, the transmitted optical power is affected by the amplitude of the displacement in the fiber and has relative insensitivity to the location of such disturbance as commonly occurs in intensity variation-based sensors [18], whereas the reflected (backscattered) is mainly affected by the location where the perturbation is applied in the fiber [28]. If the perturbation occurs close to the light source, there will be a lower backscattered power. In contrast, higher backscattered power is obtained as the distance between source and perturbation location increases. Thus, mechanical perturbation and its localization can be estimated by the analysis of both transmission and reflection optical signals, where unique relations of transmission and reflection are obtained for each location and amplitude of the mechanical perturbation [28].

As any intensity variation-based system, the TRA system is not immune to light source power fluctuations. Thus, the light source power is monitored, where the transmission and reflection optical powers are normalized as a function of the light source in order to obtain a self-compensation for optical power fluctuations [36]. The system with transmitted, reflected and reference optical powers need three photodetectors as shown in Fig. 1(b). In this case, the light source is connected to the $3 \mathrm{~dB}$ coupler TW1550R5F1 (Thorlabs, USA), which split the signal to the reference photodetector $\left(P_{r e f}\right)$ and the optical circulator 6015-3 (Thorlabs, USA). In optical circulator Port 2, there is the NPF, which has another photodetector connected to its end for the acquisition of transmitted optical power $\left(P_{\text {tran }}\right)$, whereas the Port 3 is connected to another photodetector $\left(P_{\text {back }}\right)$ in order to obtain the reflected (backscattered) optical power from the NPF. Each photodetector GT322D (Go4Fiber, Hong Kong) has a transimpedance amplifier and is connected to the microcontroller FRDM-K64F (NXP Semiconductors, Netherlands) for the signal acquisition with 16 bits resolution in the analog-to-digital conversion. The NPF is integrated in a vest with the configuration shown in Fig. 1(c). For the wearable tests, the optical components and electronics can be positioned on the back of the vest as also depicted in Fig. 1(c).

As discussed in previous works [23], the region close to the fiber end facets are the ones with the lowest sensitivity, since the gap between the transmission-reflection curves is lower in these regions. For this reason, there are two regions of about $3 \mathrm{~m}$, one at each end facet, in which no displacement is applied on the fiber. These regions are close to the optical and electronics components in the back of the garment. Hence, we guarantee that the mechanical perturbations are applied on the regions where the highest sensitivity is obtained, as shown in Fig. 1(c). In contrast with the previously reported works on the TRA technique, where the only the location of the perturbation is estimated, the goal of the proposed work is to estimate both location and amplitude of the perturbation. The mechanical perturbations are applied in predefined points on the vest, where two scenarios are considered: one off-body for the sensor's characterization and the other with the smart garment positioned on the user.

In the first scenario, i.e., characterization tests, the calibrated weights are positioned on different points as presented in Fig. 1(c), where the garment is positioned on a soft surface (polyurethane surface), resulting in different displacements on the optical fiber. The fiber displacement and its location on the fiber are obtained from the transmitted and reflected signals. The fiber displacement is illustrated in Fig. 1(c) inset. The fiber displacement is analyzed instead of the applied force, since it is the parameter that directly correlates with the optical power variations. If the same force is applied in the predefined points, but with different supporting materials, it also leads to different displacement due to the different stiffness of the support material, which provides different restrictions to the fiber displacement. It is worth to mention that it is possible to estimate the applied force or pressure on the fiber from the displacement data if the stiffness of the support material is known, using the Hooke's law to obtain the force from the stiffness and displacement. Thus, for the same applied transverse force the displacement on the fiber will be higher if it is on a polymer support than on a metal support. Considering the wearable application for mechanical perturbation assessment, the NPF is positioned at different regions of the user's body as can be seen in Fig. 1(c). In this case, the back region generally has higher stiffness than the chest or abdominal region, since they have a larger layer of soft tissue, which makes it difficult to provide a direct relation with the applied force using only the fiber displacement and without any previous on-body calibration. It is also noteworthy that the stiffness of each region also varies between subjects according to parameters such as gender and body mass. These reasons also motivate the use of the fiber displacement, since the displacement considers all the aforementioned parameters, i.e., material stiffness, applied force amplitude and position.

In the sensor system characterization, displacements ranging from $5 \mathrm{~mm}$ to $50 \mathrm{~mm}$ (in $15 \mathrm{~mm}$ steps) are applied on the fiber by positioning calibrated weights on the smart garment positioned over a polyurethane support, as shown in Fig. 1(c). The displacements are applied at 8 predefined distances (with respect to the fiber end facet) of $3.6 \mathrm{~m}, 3.8 \mathrm{~m}, 4.0 \mathrm{~m}, 4.2 \mathrm{~m}$, $4.4 \mathrm{~m}, 4.65 \mathrm{~m}, 4.9 \mathrm{~m}$ and $5.2 \mathrm{~m}$, where all the tests are performed at constant temperature conditions and five tests were performed at each displacement and location conditions. In this characterization, the technique for simultaneous assessment of perturbation location and optical fiber displacement is obtained. Figure 2 shows the schematic representation of the technique, where the signals presented are obtained considering linear behavior of both transmission and reflection responses at different displacement (considered as $\mathrm{D}_{1}$ to $\mathrm{D}_{5}$ for generalization purposes) applied at each location condition (considered as $\mathrm{L}_{1}$ to $\mathrm{L}_{9}$ for the method generalization). As shown in Fig. 2, each point on the transmission-reflection curve represents a location and displacement pair. In this case, the curves with the same slope are related to a predefined 

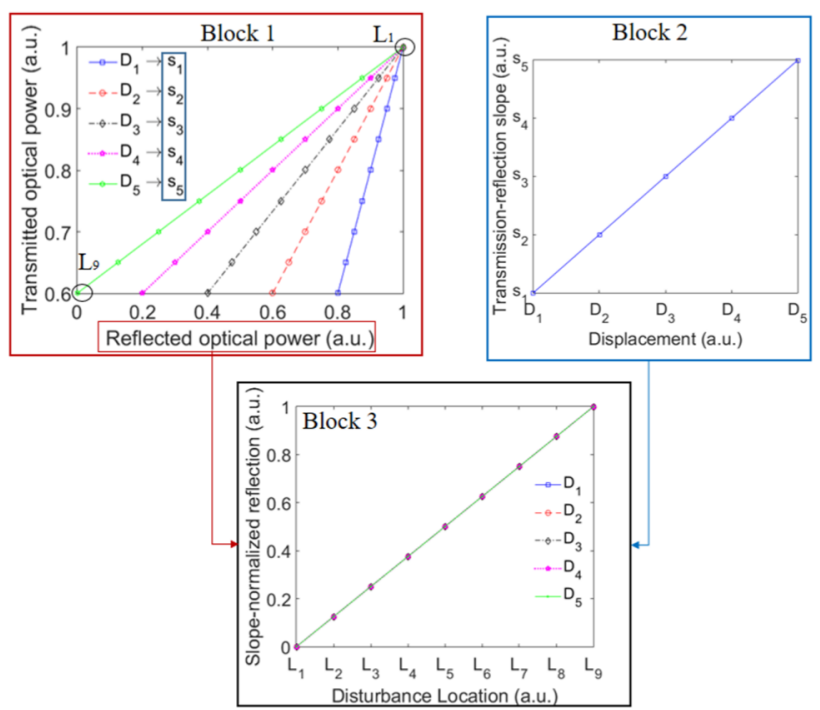

Fig. 2. Slope-assisted TRA signal processing for simultaneous assessment of disturbance location and amplitude.

displacement, whereas the points along this curve are related to the location of the disturbance.

Thus, it is possible to correlate the slope inverse of each curve $\left(s_{i}\right)$ with the fiber displacement, as shown in block 2 (see Fig. 2) in which curve slopes inverses are correlated with the applied displacement. Then, by dividing each curve to its slope inverse, the overlapping curves in block 3 are obtained. Hence, the influence of the displacement in the transmission and reflection signals is suppressed. Therefore, it is possible to obtain experimental regressions for the disturbance location by analyzing the reflection signals as a function of the disturbance location. For example, if a predefined displacement is applied in a location along the fiber, there is a transmission and a reflection optical normalized power related to the displacement amplitude and location, which appears as a point in the transmission-reflection plane shown in Block 1. In this case, this point at transmission-reflection plane is compared with the initial condition, i.e., without applied displacement, which is the pair $(1,1)$ for $L_{1}=0$ as indicated in Block 1 of Fig. 2 . The slope obtained in the comparison between the initial condition and the aforementioned point at transmission-reflection plane is used on the displacement amplitude estimation following the linear regression shown in Block 2. Then, the reflection signal is normalized as a function of the aforementioned slope (between the initial condition and the point on the transmission-reflection plane) for the displacement location estimation using the regression shown in Block 3.

After the smart garment characterization tests, on-body tests are performed. Three volunteers (two females and one male) used the garment and mechanical disturbances were applied at four predefined points in the TRA-based smart garment ( $3.6 \mathrm{~m}, 4.6 \mathrm{~m}, 4.9 \mathrm{~m}$ and $5.2 \mathrm{~m}$ ). The technique presented in Fig. 2 was used to obtain the displacement and disturbance location on each condition. In order to verify if the natural movement during the gait result in cross-sensitivity on the sensors responses, the users were also asked to walk during

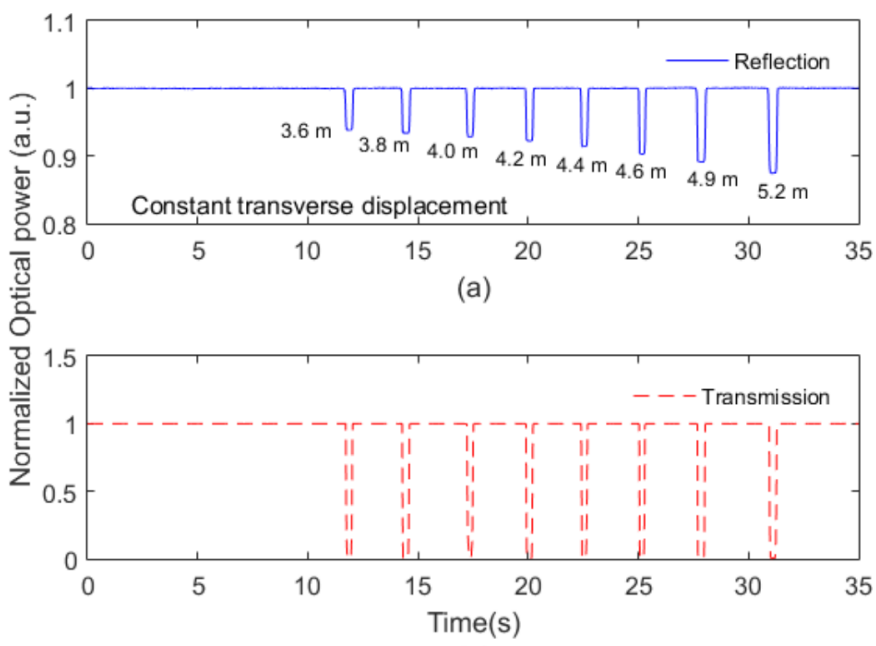

(b)

Fig. 3. (a) Reflection and (b) transmission optical normalized powers as a function of time for constant displacement $(50 \mathrm{~mm})$ applied on different locations along the optical fiber.

the tests. The mechanical disturbances were performed using the mechanical disturbance element shown in Fig. 1(c), which was positioned on the predefined location with a constant distance to the garment. Then, a $20 \mathrm{~mm}$ displacement is performed by the disturbance element with the aid of an external ruler. In order to verify if the displacement in the fiber has a correlation in the tests performed with different volunteers, the determination coefficient $\left(\mathrm{R}^{2}\right)$ between the combinations of each volunteer results was analyzed.

\section{Results and Discussion}

The transmitted and reflected normalized powers for the first test on the different locations along the NPF are shown in Fig. 3. In this test, a constant displacement of about $50 \mathrm{~mm}$ was applied at each location. Both transmission and reflection signals are normalized with respect to the reference signal acquired directly from the light source (see Fig. 1). Such large displacement results in a large reduction of the transmitted optical power, as can be seen from the red dashed curve in which the optical power is close to 0 . The results in the transmission also show a high repeatability of the sensor, where a standard deviation as low as $2.89 \times 10^{-5}$ a.u. was obtained considering all tested locations. Regarding to the reflected optical power, there is a quasi-linear decrease of the reflected power with the increase of the distance to the fiber connector (from the first location at $3.6 \mathrm{~m}$ to the last in $5.2 \mathrm{~m}$ ). These results indicate the possibility of simultaneous assessment of the displacement on the optical fiber and its location along the fiber.

As Fig. 3 showed the repeatability in all locations, the sensor repeatability at different tests is presented in Fig. 4, where the normalized reflected signals for a $5 \mathrm{~mm}$ displacement at each location are shown as a function of the distance from the connector in order to verify the sensor repeatability. Since there is no significant variation in the transmission signals (as presented in Fig. 3), only the reflected optical power is presented in Fig. 4. The sensor responses show a high 


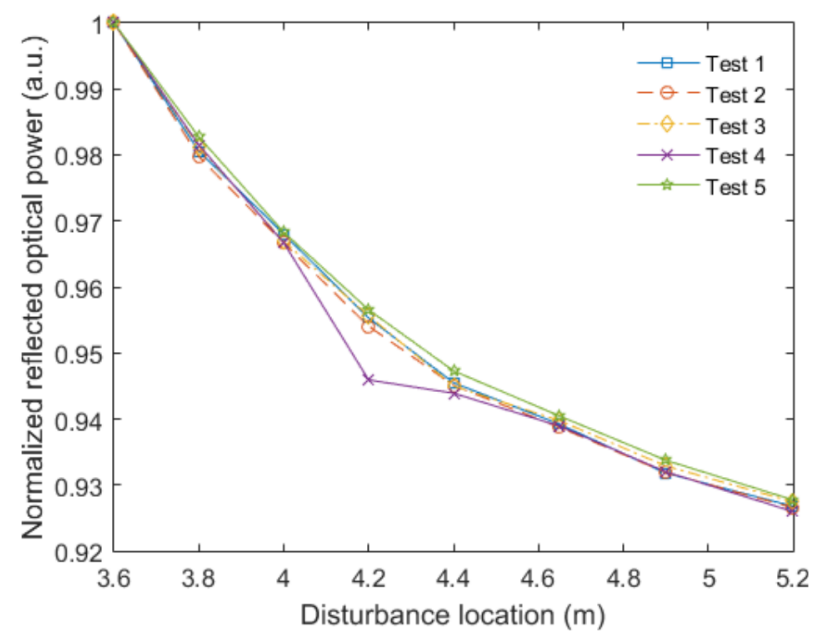

Fig. 4. Normalized reflected optical power for constant displacements applied on different locations for 5 tests.

repeatability of the sensor, where there is almost an overlap between the curves of each test. The only point with significant difference from the others is the one at $4.2 \mathrm{~m}$ for test 4 . Such variation can be related to errors in the weight positioning in the fiber, since there is a small distance between them (only $20 \mathrm{~cm}$ ). Nevertheless, all other points show a similar behavior with standard deviations close to $2.5 \times 10^{-4}$ a.u. For this reason, the results and analysis at different locations and displacements are presented as the mean of the five performed tests at each condition.

Thereafter, different displacements (from $5 \mathrm{~mm}$ to $50 \mathrm{~mm}$ in $15 \mathrm{~mm}$ steps) were applied along the NPF at predefined locations. The applied displacements lead to variations in the reflection and transmission responses of the NPF. Thus, there is a variation in the slope of the transmission-reflection curves as shown in Fig. 5(a). The comparison between the transmission-reflection curves show that there is a slope variation as a function of the applied displacement. In fact, the slope inverse of transmission-reflection curves can be used for the displacement estimation on the NPF as shown in Fig. 5(b), where a $R^{2}$ of 0.996 was found in the linear regression. Thus, the ratio between the variations of the transmission and reflection signals is used on the displacement estimation for the wearable smart garment based on TRA in NPF.

Following the procedures depicted in Fig. 2 for the simultaneous estimation of displacement amplitude and location along the optical fiber, reflection signals are divided by the curve slope inverse. Figure 6(a) shows the reflection signals divided by the curve slope inverse as a function of the distance to the connector. The results indicate similar behavior of the normalized responses even when considering the different displacements applied on the fiber. For this reason, a linear regression of the mean reflection response was applied in order to obtain the disturbance location as a function of the slopenormalized reflection response. Figure $6(\mathrm{~b})$ shows disturbance location estimation results using the linear regression presented in Fig. 6(a) (solid black line) to estimate the location at each displacement condition. In addition, the inset of Fig. 6(b)

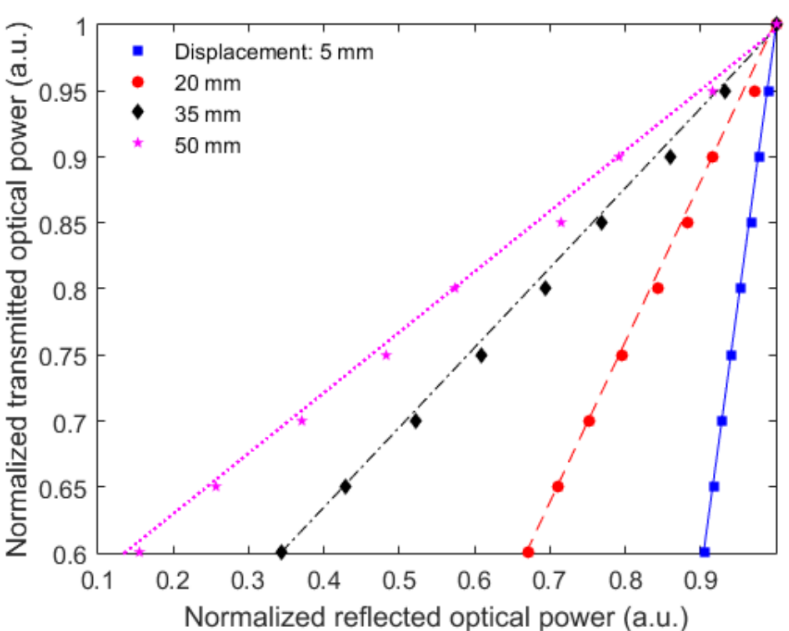

(a)

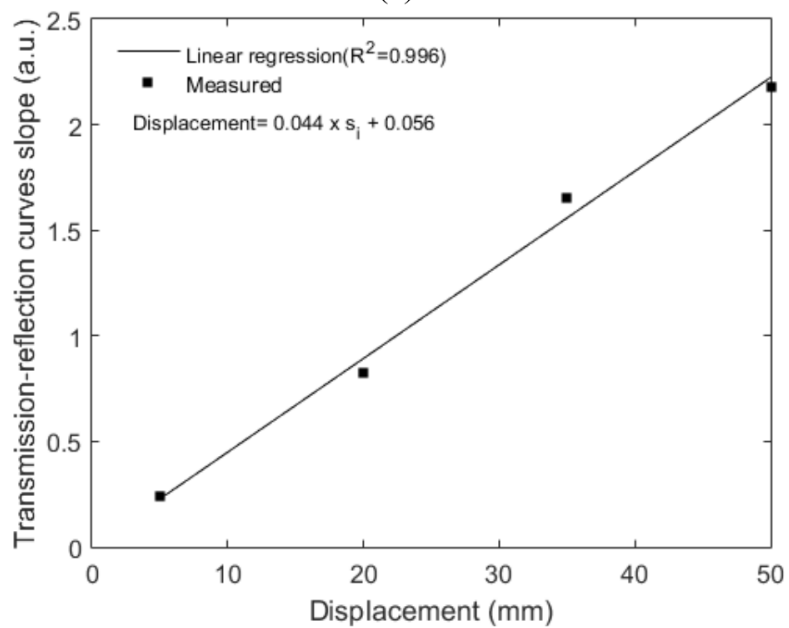

(b)

Fig. 5. (a) Transmission-reflection curves for different displacements. (b) Slope inverses of the transmission-reflection curves as a function of the fiber displacement.

shows the root mean squared error (RMSE) for each analyzed case.

The obtained results show errors generally below $80 \mathrm{~mm}$, which, considering the distance range tested, indicates a relative error of $3.7 \%$. The errors on the location estimation are related to nonlinearities in the sensors responses, as presented in Fig. 6(a), where there are deviations between the measured points (divided by the transmission-reflection curve slope inverse) and the linear regression (black solid line). Nevertheless, such results indicate the feasibility of the proposed approach on the disturbance detection in NPF, where the fiber displacement and its location are simultaneously assessed, which motivate its use on the assessment of gait perturbations.

Then, on-body tests were performed with 3 volunteers, one male (Volunteer 1) and two females (Volunteer 2 and 3). In the first analysis, the reflection signals of the smart garment are acquired in three conditions: (i) standing, (ii) under mechanical perturbations on the regions presented in Fig. 1(c) using the mechanical disturbance element and (iii) walking. Figure 7(a) shows the reflection response in these three conditions (for 


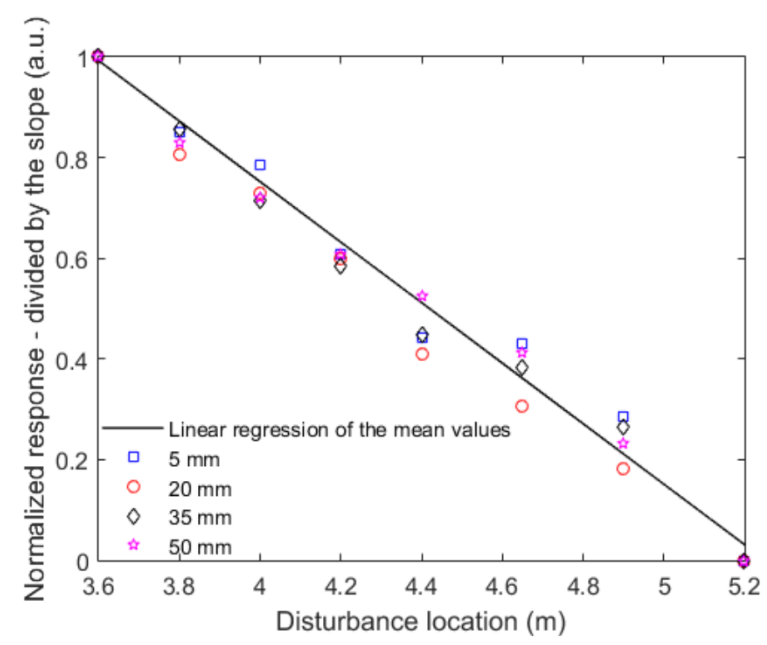

(a)

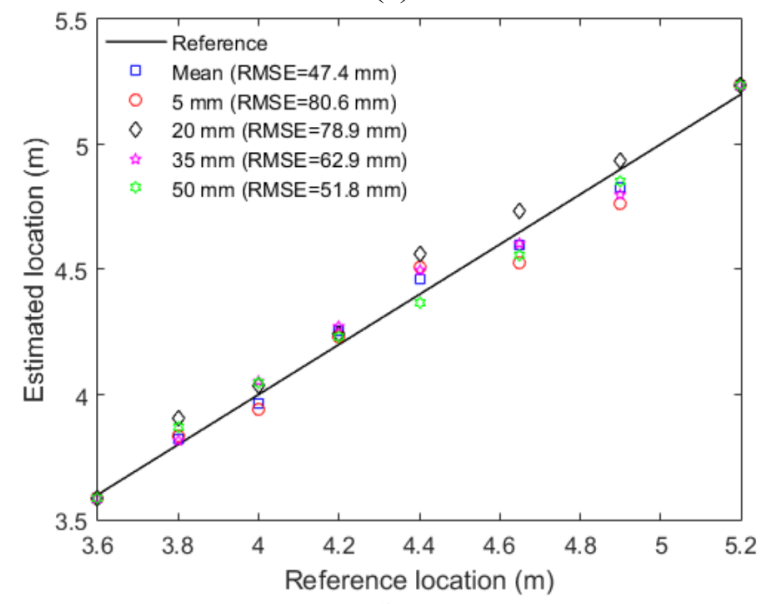

(b)

Fig. 6. (a) Slope-normalized reflected optical power as a function of the disturbance location. (b) Disturbance location estimation for each optical fiber displacement.

Volunteer 3) to verify if the proposed sensor system is able of distinguish the mechanical perturbations from signal variations that can normally occur during gait. As shown in Fig. 7(a), there are three sets of sequential mechanical perturbations on predefined points on the smart garment with significant signal variations when compared with the standing and walking cases. Therefore, the natural body movements during gait do not lead to significant cross-sensitivity in the proposed approach for mechanical perturbation assessment during. It also indicates that no further signal processing is needed to filter out the gait effects on the distributed sensor system responses.

Following cross-sensitivity analysis in which the influence of the gait natural movements on the mechanical perturbation assessment is considered negligible. The mechanical perturbations were applied on four predefined points in the TRA-based smart garment, i.e., $3.6 \mathrm{~m}, 4.6 \mathrm{~m}, 4.9 \mathrm{~m}$ and $5.2 \mathrm{~m}$ as shown in Fig. 1(c). Figure 7(b) shows the estimated fiber displacement and location of the mechanical disturbances along the garment for the tests with each volunteer. As aforementioned and shown in Fig. 1(c), a displacement of about $20 \mathrm{~mm}$ is applied on the mechanical disturbance element at each point for

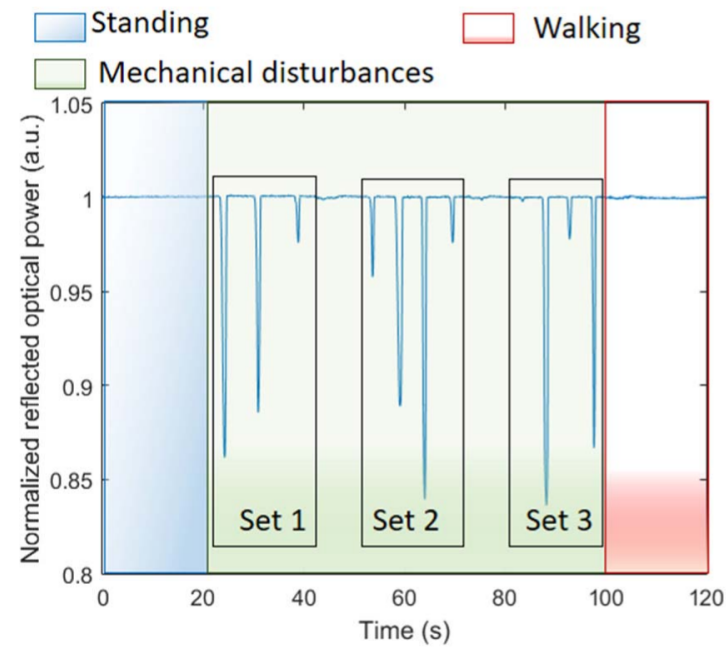

(a)
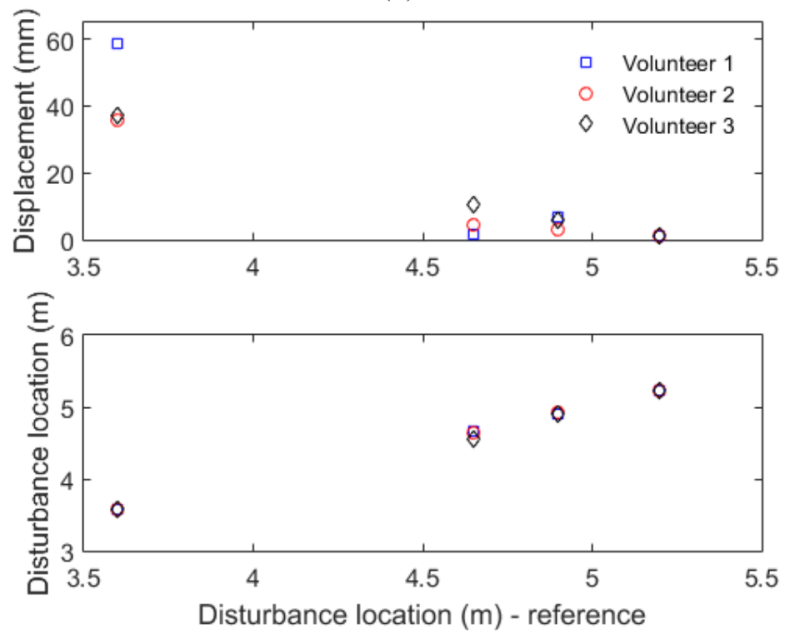

(b)

Fig. 7. (a) Normalized reflected optical power as a function of time for different conditions: standing, under mechanical disturbances and walking without disturbances. (b) Displacement and location estimations for the on-body tests.

the on-body tests. However, the tests were performed with different volunteers, where Volunteer 1 has a different gender and, thus, a different body structure, this feature explains the major differences especially in location 1 (at $3.6 \mathrm{~m}$ ), which is on the chest region. There are also lower differences in the fiber displacement on other locations, especially at the location of $5.2 \mathrm{~m}$, in the abdominal region. In this case, it is expected a correlation between samples, since similar displacements are applied on the mechanical disturbance element. For this reason, the correlation between the tests of each volunteer was verified. In this analysis, a mean $\mathrm{R}^{2}$ of 0.984 was found, indicating a high correlation in the displacement estimation in all performed tests with each volunteer, which also indicate a high repeatability of the proposed sensor system.

For the disturbance location estimation, the comparison with the reference location (obtained from measuring the fiber in the predefined displacement application points) shows a high accuracy on the location estimation, where RMSEs of about $21 \mathrm{~mm}$ and $51 \mathrm{~mm}$ were obtained in the best (for Volunteer 1) and worst cases (for Volunteer 3), respectively. 
Considering the location range in the tests $(1.6 \mathrm{~m})$, the mean RMSE (32 mm) resulted in relative errors of $2.1 \%$, which can be regarded as low errors for gait analysis and exoskeleton disturbance rejection control assessment.

It is worth noting that TRA-based sensors systems are not able of measuring multiple perturbations simultaneously. However, such limitation does not harm many applications of the proposed system. In addition to the proposed application on mechanical perturbation assessment during gait, there is also possible to apply the proposed system in smart carpets for ground reaction forces and spatiotemporal parameters assessment during gait [5], since the gait is comprised of well-defined sequential phases. Furthermore, the proposed system can be used on intrusion detection systems [37] and in impact detection matrices such as the one presented in [20]. Another possible applications for the proposed system are sensing pad in haptic feedback tasks for physical and cognitive training [38]. Nevertheless, the proposed system may be able of detecting simultaneous perturbations by modulating the light source as demonstrated in [39], where a frequency sweep is performed on the light source signal and the sensor response is also analyzed in the frequency domain with the drawback of increasing the system's cost and bulkiness.

\section{CONCLUSION}

This paper presented the development of a wearable smart garment for mechanical perturbations assessment during gait. Such novel wearable distributed optical fiber sensing system was obtained using the TRA technique, resulting in a portable and low cost system, since the sensor interrogation is achieved with only photodetectors and a microcontroller. In addition, a NPF was employed as a high scattering medium in which exceptionally high spatial is obtained in the TRA (up to three orders of magnitude higher than the one obtained with conventional SMFs). The NPF was embedded in a garment and different mechanical disturbances were applied with predefined displacements and locations. In order to simultaneously measure the fiber displacement and the perturbation location, a slope-assisted signal processing technique for TRA was proposed. In the proposed technique, the slope inverses of the transmission-reflection curves were correlated with the fiber displacement, whereas the slope-normalized reflection signal was used on a linear regression for the disturbance location estimation. The technique was verified in characterization tests, where relative errors as low as $3.7 \%$ were found on the parameters estimation. Then, on-body tests were performed and indicated a negligible cross-sensitivity between the sensor responses under transverse mechanical perturbation and on the natural gait movements. The sensor was validated in three volunteers in which high correlation $\left(\mathrm{R}^{2}\right.$ of 0.984$)$ in the displacement estimation were found between tests and the location estimation errors were as low as $21 \mathrm{~mm}$.

The results obtained in this work not only shows the feasibility of the proposed approach, but also its advantages in many applications, especially when compared with conventional electronic sensors. The proposed sensor system has negligible electromagnetic interference, which makes it suitable for its use with exoskeleton systems. In addition, the system is compact, fully portable and does not inhibit the natural movements of the user. As another important advantage, the signal processing is simple and is inherently insensitive to signal variations due to natural gait movements. Moreover, as the materials are not electrically conductive, the proposed smart garment is immune to variations due to user's sweat, which also makes it safer for the user. Therefore, the proposed smart garment can be readily employed on gait analysis under mechanical perturbation as well as on the assessment of exoskeleton disturbance rejection control. The presented signal processing technique was focused on the physical effects and mechanisms on the signal. However, it is noteworthy that machine learning techniques such as support vector machines and artificial neural networks can further improve the sensor accuracy and will be investigated in future works. Another future work is the analysis and correlation between the applied displacement and force at different stiffness conditions of the substrate.

\section{ACKNOWLEDGMENT}

The authors acknowledge S. Trzesien and M. Ude (INPHYNI, Nice, France) for the fabrication of the fiber.

\section{REFERENCES}

[1] G. R. Thomas and A. A. Khatibi, "Durability of structural health monitoring systems under impact loading," Procedia Eng., vol. 188 , pp. 340-347, Jan. 2017.

[2] C. Gandiolle and S. Fouvry, "FEM modeling of crack nucleation and crack propagation fretting fatigue maps: Plasticity effect," Wear, vols. 330-331, pp. 136-144, May 2015.

[3] A. G. Leal-Junior, C. R. Díaz, C. Marques, M. J. Pontes, and A. Frizera, "3D-printed POF insole: Development and applications of a lowcost, highly customizable device for plantar pressure and ground reaction forces monitoring," Opt. Laser Technol., vol. 116, pp. 256-264, Aug. 2019.

[4] A. G. Leal-Junior et al., "Polymer optical fiber strain gauge for humanrobot interaction forces assessment on an active knee orthosis," Opt. Fiber Technol., vol. 41, pp. 205-211, Mar. 2018.

[5] L. M. Avellar, A. G. Leal-Junior, C. A. R. Diaz, C. Marques, and A. Frizera, "POF smart carpet: A multiplexed polymer optical fiberembedded smart carpet for gait analysis," Sensors, vol. 19, no. 15, p. 3356, Jul. 2019

[6] D. A. Winter, "Human balance and posture control during standing and walking," Gait Posture, vol. 3, pp. 193-214, Dec. 1995.

[7] F. Madehkhaksar, J. Klenk, K. Sczuka, K. Gordt, I. Melzer, and M. Schwenk, "The effects of unexpected mechanical perturbations during treadmill walking on spatiotemporal gait parameters, and the dynamic stability measures by which to quantify postural response," Plos One, vol. 13, no. 4, pp. 1-15, 2018, doi: 10.1371/journal.pone.0195902.

[8] J. Mueller, T. Engel, S. Mueller, S. Kopinski, H. Baur, and F. Mayer, "Neuromuscular response of the trunk to sudden gait disturbances: Forward vs. backward perturbation," J. Electromyograp. Kinesiol., vol. 30, pp. 168-176, Oct. 2016.

[9] W. Huo, S. Mohammed, J. C. Moreno, and Y. Amirat, "Lower limb wearable robots for assistance and rehabilitation: A state of the art," IEEE Syst. J., vol. 10, no. 3, pp. 1068-1081, Sep. 2016.

[10] C. Walsh, "Human-in-the-loop development of soft wearable robots," Nature Rev. Mater, vol. 3, no. 6, pp. 78-80, Jun. 2018, doi: 10.1038/s41578-018-0011-1.

[11] P. Tropea, F. Aprigliano, D. Martelli, A. Parri, and M. Cortese, "An ecologically-controlled exoskeleton can improve balance recovery after slippage," Sci. Rep., vol. 2016, pp. 1-10, Dec. 2017.

[12] M. Cianchetti, C. Laschi, A. Menciassi, and P. Dario, "Biomedical applications of soft robotics," Nature Rev. Mater., vol. 3, no. 6, pp. 143-153, Jun. 2018.

[13] Y. Long, Z. Du, L. Cong, W. Wang, Z. Zhang, and W. Dong, "Active disturbance rejection control based human gait tracking for lower extremity rehabilitation exoskeleton," ISA Trans., vol. 67, pp. 389-397, Mar. 2017. 
[14] L. Capineri, "Resistive sensors with smart textiles for wearable technology: From fabrication processes to integration with electronics," Procedia Eng., vol. 87, pp. 724-727, Jan. 2014.

[15] C. Gonçalves, A. F. da Silva, J. Gomes, and R. Simoes, "Wearable e-textile technologies: A review on sensors, actuators and control elements," Inventions, vol. 3, no. 1, pp. 1-13, 2018.

[16] A. G. Leal-Junior, A. Frizera-Neto, M. J. Pontes, and T. R. Botelho, "Hysteresis compensation technique applied to polymer optical fiber curvature sensor for lower limb exoskeletons," Meas. Sci. Technol. vol. 28 , no. 12 , Dec. 2017 , Art. no. 125103.

[17] P. Roriz, O. Frazão, A. B. Lobo-Ribeiro, J. L. Santos, and J. A. Simões, "Review of fiber-optic pressure sensors for biomedical and biomechanical applications," J. Biomed. Opt., vol. 18, no. 5, p. 50903, 2013.

[18] A. G. Leal-Junior, C. A. R. Diaz, L. M. Avellar, M. J. Pontes, C. Marques, and A. Frizera, "Polymer optical fiber sensors in healthcare applications: A comprehensive review," Sensors, vol. 19, no. 14, p. 3156 , Jul. 2019.

[19] Z. Ding et al., "Distributed optical fiber sensors based on optical frequency domain reflectometry: A review," Sensors, vol. 18, no. 4, p. 1072 , Apr. 2018.

[20] P. Shrestha, J.-H. Kim, Y. Park, and C.-G. Kim, "Impact localization on composite structure using FBG sensors and novel impact localization technique based on error outliers," Composite Struct., vol. 142, pp. 263-271, May 2016.

[21] A. G. Leal-Junior, V. Campos, C. Díaz, R. M. Andrade, A. Frizera, and C. Marques, "A machine learning approach for simultaneous measurement of magnetic field position and intensity with fiber Bragg grating and magnetorheological fluid," Opt. Fiber Technol., vol. 56, May 2020, Art. no. 102184.

[22] C. A. R. Diaz et al., "Perrogator: A portable energy-efficient interrogator for dynamic monitoring of wavelength-based sensors in wearable applications," Sensors, vol. 19, no. 13, p. 2962, Jul. 2019.

[23] V. V. Spirin, "Transmission-Reflection analysis for localization of temporally successive multipoint perturbations in a distributed fiber-optic loss sensor based on Rayleigh backscattering," Appl. Opt., vol. 42, no. 7, pp. 1175-1181, 2003.

[24] V. V. Spirin, F. J. Mendieta, S. V. Miridonov, M. G. Shlyagin, A. A. Chtcherbakov, and P. L. Swart, "Localization of a loss-inducing perturbation with variable accuracy along a test fiber using transmissionreflection analysis," IEEE Photon. Technol. Lett., vol. 16, no. 2, pp. 2003-2005, Feb. 2004.

[25] R. M. López, V. V. Spirin, S. V. Miridonov, M. G. Shlyagin, G. Beltrán, and E. A. Kuzin, "Fiber optic distributed sensor for hydrocarbon leak localization based on transmission/reflection measurement," Opt. Laser Technol., vol. 34, no. 6, pp. 465-469, Sep. 2002
[26] V. V. Spirin, M. G. Shlyagin, S. V. Miridonov, and P. L. Swart, "Alarmcondition detection and localization using Rayleigh scattering for a fiberoptic bending sensor with an unmodulated light source," Opt. Commun., vol. 205, nos. 1-3, pp. 37-41, Apr. 2002.

[27] W. Blanc et al., "Compositional changes at the early stages of nanoparticles growth in glasses," J. Phys. Chem. C, vol. 123, no. 47, pp. 29008-29014, Nov. 2019.

[28] M. Silveira et al., "Transmission-reflection analysis in high scattering optical fibers: A comparison with single-mode optical fiber," Opt. Fiber Technol., vol. 58, Sep. 2020, Art. no. 102303.

[29] A. Beisenova, A. Issatayeva, S. Korganbayev, C. Molardi, W. Blanc, and D. Tosi, "Simultaneous distributed sensing on multiple $\mathrm{MgO}$-doped high scattering fibers by means of scattering-level multiplexing," J. Lightw. Technol., vol. 37, no. 13, pp. 3413-3421, Jul. 1, 2019.

[30] A. Beisenova, A. Issatayeva, I. Iordachita, W. Blanc, C. Molardi, and D. Tosi, "Distributed fiber optics 3D shape sensing by means of high scattering NP-doped fibers simultaneous spatial multiplexing," Opt. Express, vol. 27, no. 16, p. 22074, 2019.

[31] C. Molardi et al., "Fiber Bragg grating (FBG) sensors in a highscattering optical fiber doped with $\mathrm{MgO}$ nanoparticles for polarizationdependent temperature sensing," Appl. Sci., vol. 9, no. 15, p. 3107, Aug. 2019.

[32] M. V. J. Lupi and F. P. M. Cabi, "Fiber-draw-induced elongation and break-up of particles inside the core of a silica-based optical fiber," J. Amer. Ceram. Soc., vol. 100, no. 5, pp. 1814-1819, Jul. 2017.

[33] D. Tosi, C. Molardi, W. Blanc, T. Paix, P. Antunes, and C. Marques, "Multiplexing (SLMux) in distributed fiber-optic backscatter reflectometry physical sensors," Sensors, vol. 20, no. 9, p. 2595, 2020.

[34] D. Tosi, C. Molardi, and W. Blanc, "Rayleigh scattering characterization of a low-loss $\mathrm{MgO}$-based nanoparticle-doped optical fiber for distributed sensing," Opt. Laser Technol., vol. 133, Jan. 2021, Art. no. 106523.

[35] A. G. Leal Junior, A. Frizera, and M. J. Pontes, "Analytical model for a polymer optical fiber under dynamic bending," Opt. Laser Technol., vol. 93, pp. 92-98, Aug. 2017.

[36] L. Bilro, N. Alberto, J. L. Pinto, and R. Nogueira, "Optical sensors based on plastic fibers," Sensors, vol. 12, no. 9, pp. 12184-12207, Sep. 2012.

[37] A. Catalano, F. A. Bruno, C. Galliano, M. Pisco, G. V. Persiano, A. Cutolo, and A. Cusano, "An optical fiber intrusion detection system for railway security," Sens. Actuators A, Phys., vol. 253, pp. 91-100, May 2018.

[38] M. Zhou, D. B. Jones, S. D. Schwaitzberg, and C. G. L. Cao, "Role of haptic feedback and cognitive load in surgical skill acquisition," in Proc. Hum. Factors Ergonom. Soc. Annu. Meeting, Oct. 2007, vol. 51, no. 11 , pp. 631-635.

[39] C. A. Rodríguez, M. R. N. Ribeiro, A. Frizera, and M. J. Pontes, "Interrogation of optical fiber based on the fusion of OFDR and TRA techniques," Opt. Quantum Electron., vol. 48, no. 4, p. 230, Apr. 2016. 